\title{
Switching via quantum activation: A parametrically modulated oscillator
}

\author{
M. Marthaler and M. I. Dykman \\ Department of Physics and Astronomy, Michigan State University, East Lansing, MI 48824, USA
}

(Dated: March 6, 2018)

\begin{abstract}
We study switching between period-two states of an underdamped quantum oscillator modulated at nearly twice its natural frequency. For all temperatures and parameter values switching occurs via quantum activation: it is determined by diffusion over oscillator quasienergy, provided the relaxation rate exceeds the rate of interstate tunneling. The diffusion has quantum origin and accompanies relaxation to the stable state. We find the semiclassical distribution over quasienergy. For $T=0$, where the system has detailed balance, this distribution differs from the distribution for $T \rightarrow 0$; the $T=0$ distribution is also destroyed by small dephasing of the oscillator. The characteristic quantum activation energy of switching displays a typical dependence on temperature and scaling behavior near the bifurcation point where period doubling occurs.
\end{abstract}

PACS numbers: 03.65.Yz, 05.60.Gg, 05.70.Ln, 74.50.+r

\section{INTRODUCTION}

Switching between coexisting stable states underlies many phenomena in physics, from diffusion in solids to protein folding. For classical systems in thermal equilibrium switching is often described by the activation law, with the switching probability being $W \propto \exp (-\Delta U / k T)$, where $\Delta U$ is the activation energy. As temperature is decreased, quantum fluctuations become more and more important, and below a certain crossover temperature switching occurs via tunneling [1, 2, 3]. The behavior of systems away from thermal equilibrium is far more complicated. Still, for classical systems switching is often described by an activation type law, with the temperature replaced by the characteristic intensity of the noise that leads to fluctuations [4, 5, 6, 7, 8, 9, 10, 11, 12. Quantum nonequilibrium systems can also switch via tunneling between classically accessible regions of their phase space [13, 14, 15, 16].

In addition to classical activation and quantum tunneling, nonequilibrium systems have another somewhat counterintuitive mechanism of transitions between stable states. We call this mechanism quantum activation and study it in the present paper. It describes escape from a metastable state due to quantum fluctuations that accompany relaxation of the system [17]. These fluctuations lead to diffusion away from the metastable state and, ultimately, to transitions over the classical "barrier", that is, the boundary of the basin of attraction to the metastable state.

We study quantum activation for periodically modulated systems. Switching mechanisms for such systems are shown schematically in Fig. 11 where $g(Q)$ is the effective potential of the system in the rotating frame. This figure describes, in particular, a nonlinear oscillator studied in the present paper, which displays period doubling when its frequency is periodically modulated in time. The energy of periodically modulated systems is not conserved. Instead they are characterized by quasienergy $\epsilon$. It gives the change of the wave function $\psi_{\epsilon}(t)$ when time is incremented by the modulation pe- $\operatorname{riod} \tau_{F}, \psi_{\epsilon}\left(t+\tau_{F}\right)=\exp \left(-i \epsilon \tau_{F} / \hbar\right) \psi_{\epsilon}(t)$, and is defined modulo $2 \pi \hbar / \tau_{F}$ für fór.

Coupling to a thermal reservoir leads to transitions between the states of the system. For $T=0$ the transitions are accompanied by the creation of excitations in the thermal reservoir. The energy of the system decreases in each transition. However, the quasienergy may decrease or increase, albeit with different probabilities $W_{\uparrow}$ and $W_{\downarrow}$. This effect has quantum origin. It is due to the functions $\psi_{\epsilon}$ being superpositions of the eigenfunctions $|N\rangle$ of the energy operator of the system in the absence of modulation. Therefore bath-induced transitions down in energy $|N\rangle \rightarrow|N-1\rangle$ lead to transitions $\psi_{\epsilon} \rightarrow \psi_{\epsilon^{\prime}}$

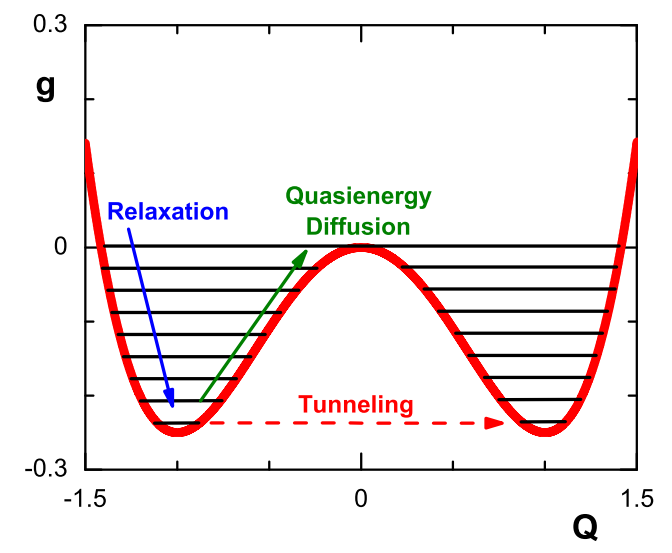

FIG. 1: (Color online) The effective double-well potential $g(Q)$ of a parametrically modulated oscillator. Sketched are scaled period-two quasienergy levels (see Sec. II) in the neglect of interwell tunneling. The minima of $g$ correspond to classically stable states of period two motion. The arrows indicate relaxation, diffusion over quasienergy away from the minima, and interwell tunneling. The effective Hamiltonian $g(P, Q)$ is defined by Eq. (6), and $g(Q) \equiv g(P=0, Q)$; the figure refers to $\mu=0$ in Eq. (6). 
with $\epsilon^{\prime} \lessgtr \epsilon$. The values of $W_{\uparrow}, W_{\downarrow}$ for the latter transitions are determined by the appropriate overlap integrals and depend on $\epsilon, \epsilon^{\prime}$.

More probable transitions determine in which direction, with respect to quasienergy, the system will most likely move. Such motion corresponds to relaxation over quasienergy. Fig. 1 refers to the case $W_{\downarrow}>W_{\uparrow}$. In this case relaxation corresponds to quasienergy decrease. The minima of $g(Q)$ are the classical stable states. However, quantum transitions in which quasienergy increases have a nonzero probability even for $T=0$. Because this probability is less than $W_{\downarrow}$, such transitions lead to diffusion over quasienergy $\epsilon$ away from the minima of $g(Q)$. In turn, the diffusion leads to a finite-width distribution over $\epsilon$ and ultimately to activated-type overbarrier transitions between the wells in Fig. 1. In fact, discussed in this paper and sketched in Fig. 1 1 are periodtwo quasienergy states, with quasienergy $\epsilon$ defined by the condition $\tilde{\psi}_{\epsilon}\left(t+2 \tau_{F}\right)=\exp \left(-2 i \epsilon \tau_{F} / \hbar\right) \tilde{\psi}_{\epsilon}(t)$. They are more convenient for the present problem; their relation to the standard quasienergy states is explained in Sec. II

Of interest for the problem of switching is the semiclassical situation where the basins of attraction to the metastable states (the wells in Fig. 1) have a large number $N$ of localized states. In this case the rate of tunneling decay is exponentially small. The activation rate is also exponentially small since it is determined by the ratio of transition probabilities $W_{\uparrow} / W_{\downarrow}$ raised to the power $N$. Both the tunneling and activation exponents are $\propto N$ for the situation sketched in Fig. 1. Indeed, the tunneling exponent is given by the action, in the units of $\hbar$, for classical motion in the inverted effective potential $-g(Q)$ from one maximum of $-g(Q)$ to the other. It is easy to see that this action is of order $N$, unless $g(Q)$ has a special form. Therefore for $N \gg 1$ the activation exponent is either much larger or much smaller than the tunneling exponent.

In this paper we develop a theory of the statistical distribution and consider switching of a parametrically modulated quantum oscillator. We show that, irrespective of temperature, the activation exponent is smaller than the tunneling exponent. Therefore switching always occurs via activation, not tunneling, as long as the relaxation rate exceeds the tunneling rate.

We study a nonlinear oscillator with frequency modulated at nearly twice the natural frequency $\omega_{0}$. When the modulation is sufficiently strong, the oscillator has two stable vibrational states with periods $2 \tau_{F} \approx 2 \pi / \omega_{0}$. These states differ in phase by $\pi$ but otherwise are identical [18]. They correspond to the minima of the effective potential in Fig. 1] The lowest quantized states in Fig. 1 are squeezed. Squeezing in a parametric oscillator has attracted interest in many areas, from quantum optics [19, 20, 21] to phonons [22], microcantilevers [23] and electrons and ions in Penning traps [24]. Recent progress in systems based on Josephson junctions and nanoelectromechanical systems [25, 26, 27, 28] makes it possible to study squeezed states in a well controlled and versatile

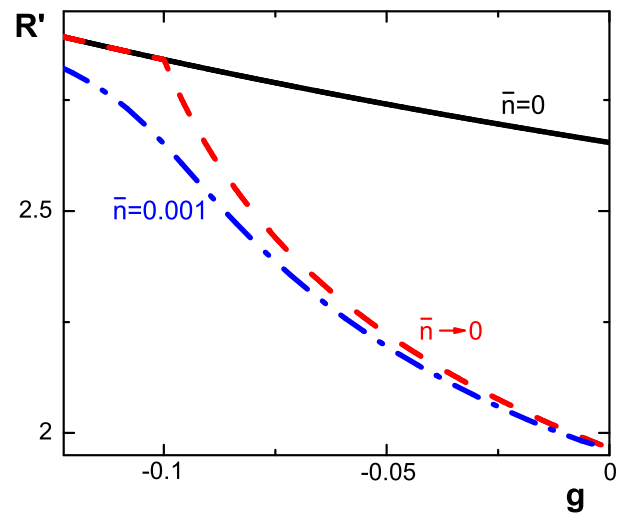

FIG. 2: (Color online) The scaled inverse temperature $R^{\prime} \equiv$ $d R / d g$ of the distribution over scaled period-two quasienergy $g$ of a parametrically modulated oscillator for different oscillator Planck numbers $\bar{n}$ in the limit of a large number of intrawell quasienergy states. The figure refers to $\mu=-0.3$.

environment. Both classical and quantum fluctuations can be investigated and the nature of switching between the states can be explored. The results can be further used in quantum measurements for quantum computing, as in the case of switching between coexisting states of a resonantly driven oscillator [25].

The probability distribution and interstate transitions of a parametrically modulated oscillator have attracted considerable attention. Much theoretical work has been done for models where fluctuations satisfy the detailed balance condition either in the classical limit [29] or for $T=0[30,31,32,33,34]$. Generally this condition does not hold in systems away from equilibrium. In particular a classical nonlinear parametric oscillator does not have detailed balance. Switching of such an oscillator was studied experimentally for electrons in Penning traps [35, 36]. The measured switching rate [36] agreed quantitatively with the theory [37.

A quantum parametric oscillator also does not have detailed balance in the general case. The results presented below show that breaking the special condition where detailed balance holds leads to a sharp change of the statistical distribution and the switching rate. This change occurs already for an infinitesimally small deviation from detailed balance, in the semiclassical limit. The fragility of the detailed balance solution is previewed in Fig. 2. This figure shows the effective inverse temperature of the intrawell distribution over the scaled periodtwo quasienergy $g$. The function $\lambda^{-1} R(g)$ is the exponent of the distribution $\lambda^{-1} R\left(g_{n}\right)=-\ln \rho_{n}$, where $\rho_{n}$ is the population of an $n$-th state and $\lambda \propto 1 / N$ is the scaled Planck constant defined in Eq. (4) below. The effective inverse temperature $\lambda^{-1} d R / d g$ depends on $g$ and differs from the inverse temperature of the bath $T^{-1}$. The 


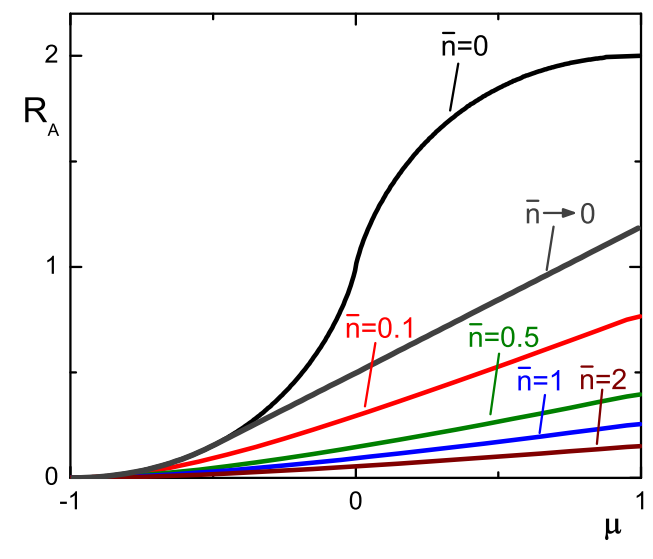

FIG. 3: (Color online) Quantum activation energy of a transition between the stable states of period two vibrations of a modulated oscillator (a phase-flip transition). The transition probability is $W_{\mathrm{sw}} \propto \exp \left(-R_{A} / \lambda\right)$, where $\lambda$, Eq. (4), is the effective Planck constant. The scaled switching exponent $R_{A}$ is plotted as a function of the single scaled parameter $\mu$ that controls oscillator dynamics, Eq. (8), for different values of the oscillator Planck number $\bar{n}$.

$T=\bar{n}=0$ result for $d R / d g$ is obtained from the solution with detailed balance (here $\bar{n}=\left[\exp \left(\hbar \omega_{0} / k T\right)-1\right]^{-1}$ is the Planck number of the oscillator). It is seen from Fig. 2 that the $T \rightarrow 0(\bar{n} \rightarrow 0)$ limit of the solution without detailed balance does not go over into the $T=0$ result.

The effective quantum activation energy $R_{A} \approx$ $-\lambda \log W_{\mathrm{sw}}$, which gives the exponent of the switching rate $W_{\mathrm{sw}}$, is shown in Fig. 3. It is equal to $R_{A}=$ $R(0)-R\left(g_{\min }\right)$, where $g=0$ and $g=g_{\text {min }}$ are, respectively, the values of $g(Q)$ at the top and the minimum of the wells in Fig. 1. The $\bar{n}=0$ detailed balance value for $R_{A}$ strongly differs from the $\bar{n} \rightarrow 0$ value in a broad range of the control parameter $\mu$ that characterizes the detuning of the modulation frequency from $2 \omega_{0}$, see Eq. (8) below. It is also seen from Fig. 3 that the quantum activation energy $R_{A}$ decreases with increasing temperature. Ultimately when the Planck number of the oscillator becomes large, $\bar{n} \gg 1$, we have $R_{A} \propto T^{-1}$, the law of thermal activation, and the results coincide with the results [37] obtained by a different method.

In Sec. II we obtain an effective Hamiltonian that describes the oscillator dynamics in the rotating frame. We describe the classical dynamics and calculate semiclassical matrix elements of the coordinate and momentum. In Sec. III we derive the balance equation for the distribution over quasienergy levels. This distribution is found in the eikonal approximation in Sec. IV] Its explicit form is obtained in several physically important limiting cases in Sec. V In Sec. VI the probability of interstate switching due to quantum activation is analyzed. In Sec. VII it is shown that the distribution obtained for $T=0$, where the system has detailed balance, is fragile: in the limit of a large number of intrawell states it differs from the distribution for $T \rightarrow 0$. It is also dramatically changed by even weak dephasing due to external noise. In Sec. VIII we discuss tunneling between the coexisting states of period two vibrations and show that interstate switching occurs via quantum activation rather than tunneling, if the relaxation rate exceeds the tunneling rate. In Sec. IX we summarize the model, the approximations, and the major results.

\section{DYNAMICS OF THE PARAMETRIC OSCILLATOR}

\section{A. The Hamiltonian in the rotating frame}

We will study quantum fluctuations and interstate switching using an important model of a bistable system, a parametric oscillator. The Hamiltonian of the oscillator has a simple form

$$
H_{0}=\frac{1}{2} p^{2}+\frac{1}{2} q^{2}\left(\omega_{0}^{2}+F \cos \left(\omega_{F} t\right)\right)+\frac{1}{4} \gamma q^{4} .
$$

We will assume that the modulation frequency $\omega_{F}$ is close to twice the frequency of small amplitude vibrations $\omega_{0}$, and that the driving force $F$ is not large, so that the oscillator nonlinearity remains small,

$$
\begin{array}{r}
\delta \omega=\frac{1}{2} \omega_{F}-\omega_{0}, \quad|\delta \omega| \ll \omega_{0}, \\
F \ll \omega_{0}^{2}, \quad|\gamma|\left\langle q^{2}\right\rangle \ll \omega_{0}^{2} .
\end{array}
$$

Here, $\left\langle q^{2}\right\rangle$ is the mean squared oscillator displacement; in what follows for concreteness we set $\gamma>0$.

We will change to the rotating frame using the canonical transformation $U(t)=\exp \left(-i \hat{a}^{\dagger} \hat{a} \omega_{F} t / 2\right)$, where $\hat{a}^{\dagger}$ and $\hat{a}$ are the raising and lowering operators, $\hat{a}=\left(\hbar \omega_{F}\right)^{-1 / 2}\left(i p+\omega_{F} q / 2\right)$. It is convenient to introduce the dimensionless coordinate $\mathrm{Q}$ and momentum $\mathrm{P}$,

$$
\begin{array}{r}
U^{\dagger}(t) q U(t)=C\left[P \cos \left(\omega_{F} t / 2\right)-Q \sin \left(\omega_{F} t / 2\right)\right],(3) \\
U^{\dagger}(t) p U(t)=-C \frac{\omega_{F}}{2}\left[P \sin \left(\omega_{F} t / 2\right)+Q \cos \left(\omega_{F} t / 2\right)\right],
\end{array}
$$

where $C=(2 F / 3 \gamma)^{1 / 2}$. The commutation relation between $P$ and $Q$ has the form

$$
[P, Q]=-i \lambda, \quad \lambda=3 \gamma \hbar / F \omega_{F} .
$$

The dimensionless parameter $\lambda$ will play the role of $\hbar$ in the quantum dynamics in the rotating frame. This dynamics is determined by the Hamiltonian

$$
\tilde{H}_{0}=U^{\dagger} H U-i \hbar U^{\dagger} \dot{U} \approx \frac{F^{2}}{6 \gamma} \hat{g}
$$


with

$$
\begin{aligned}
\hat{g} & \equiv g(P, Q) \\
& =\frac{1}{4}\left(P^{2}+Q^{2}\right)^{2}+\frac{1}{2}(1-\mu) P^{2}-\frac{1}{2}(1+\mu) Q^{2} .
\end{aligned}
$$

Here we used the rotating wave approximation and disregarded fast oscillating terms $\propto \exp \left( \pm i n \omega_{F} t\right), n \geq 1$. As a result $\tilde{H}_{0}$ is independent of time.

In the "slow" dimensionless time

$$
\tau=t F / 2 \omega_{F}
$$

the Schrödinger equation has the form $i \lambda d \psi / d \tau=\hat{g} \psi$. The effective Hamiltonian $\hat{g}$, Eq. (6), depends on one parameter

$$
\mu=2 \omega_{F} \delta \omega / F
$$

For $\mu>-1$, the function $g(P, Q)$ has two minima. They are located at $P=0, Q= \pm(\mu+1)^{1 / 2}$, and $g_{\min }=$ $-(\mu+1)^{2} / 4$. For $\mu \leq 1$ the minima are separated by a saddle at $P=Q=0$, as shown in Fig. 4. When friction is taken into account, the minima become stable states of the parametrically excited vibrations in the classical limit [18, 37] [we note that the function $G$ in Ref. 37 is similar to $g(P, Q)$, but has opposite sign].

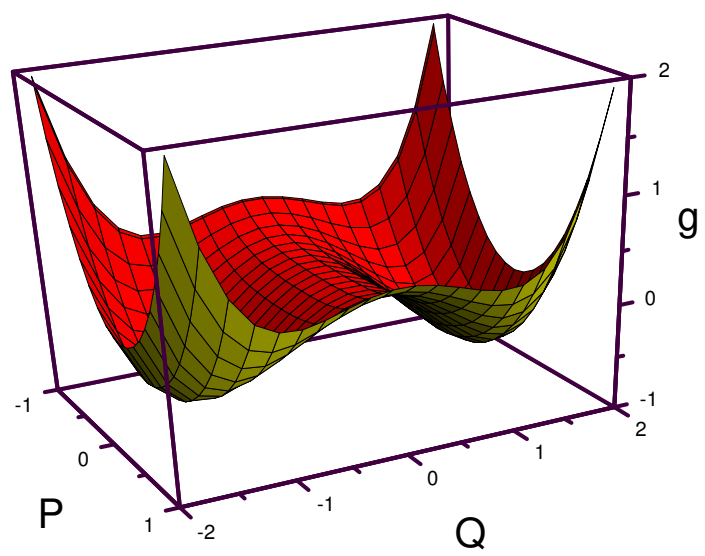

FIG. 4: (Color online) The scaled effective Hamiltonian of the oscillator in the rotating frame $g(P, Q)$, Eq. (6), for $\mu=$ 0.2 . The minima of $g(P, Q)$ correspond to the stable states of period two vibrations.

The function $g(P, Q)$ is symmetric, $g(P, Q)=$ $g(-P,-Q)$. This is a consequence of the time translation symmetry. The sign change $(P, Q) \rightarrow(-P,-Q)$ corresponds to the shift in time by the modulation pe$\operatorname{riod} t \rightarrow t+2 \pi / \omega_{F}$, see Eq. (3). In contrast to the standard Hamiltonian of a nonrelativistic particle, the function $g(P, Q)$ does not have the form of a sum of kinetic and potential energies, and moreover, it is quartic in momentum $\mathrm{P}$. This structure results from switching to the rotating frame. It leads to a significant modification of quantum dynamics, in particular tunneling, compared to the conventional case of a particle in a static potential.

\section{B. Quasienergy spectrum and classical motion}

\section{Period-two quasienergy states}

The quasienergies of the parametric oscillator $\epsilon_{m}$ are determined by the eigenvalues $g_{m}$ of the operator $\hat{g}=g(P, Q)$, with an accuracy to small corrections $\sim F / \omega_{F}^{2}$. They can be found from the Schrödinger equation $\hat{g}|m\rangle^{(0)}=g_{m}|m\rangle^{(0)}$. Because of the symmetry of $\hat{g}$ the exact eigenfunctions $|m\rangle^{(0)}$ are either symmetric or antisymmetric in $Q$.

The eigenstates $|m\rangle^{(0)}$ give the Floquet states of the parametric oscillator $\psi_{\epsilon}\left(t+\tau_{F}\right)=\exp \left(-i \epsilon \tau_{F} / \hbar\right) \psi_{\epsilon}(t)$. From the form of the unitary operator $U(t)=$ $\exp \left(-i \hat{a}^{\dagger} \hat{a} \omega_{F} t / 2\right)$ it is clear that $U\left(\tau_{F}\right)|m\rangle^{(0)}= \pm|m\rangle^{(0)}$, with "+" for symmetric and "_" for antisymmetric states $|m\rangle^{(0)}$. Therefore we have $\epsilon_{m}=\left(F^{2} / 6 \gamma\right) g_{m}$ for symmetric and $\epsilon_{m}=\left(F^{2} / 6 \gamma\right) g_{m}+\left(\hbar \omega_{F} / 2\right)$ for antisymmetric states.

It is convenient to define period-two quasienergy by the condition $\tilde{\psi}_{\epsilon}\left(t+2 \tau_{F}\right)=\exp \left(-2 i \epsilon \tau_{F} / \hbar\right) \tilde{\psi}_{\epsilon}(t)$. In this case the relation $\epsilon_{m}=\left(F^{2} / 6 \gamma\right) g_{m}$ holds for both symmetric and antisymmetric states. At the same time, there is simple one to one correspondence with the standard quasienergy states. Period-two quasienergy states are particularly convenient for describing intrawell states $|n\rangle$ for small tunneling between the wells of $g(P, Q)$. Such states are combinations of symmetric and antisymmetric states $|m\rangle^{(0)}$. For this reason we use period-two quasienergies throughout the paper.

\section{Classical intrawell motion}

We will study the intrawell states $|n\rangle$ in the semiclassical approximation. The classical equations of motion

$$
\frac{d Q}{d \tau}=\partial_{P} g, \quad \frac{d P}{d \tau}=-\partial_{Q} g
$$

can be explicitly solved in terms of the Jacobi elliptic functions. The solution is given in Appendix A. There are two classical trajectories for each $g<0$, one per well of $g(P, Q)$ in Fig. 4. They are inversely symmetrical in phase space and double periodic in time,

$$
Q\left(\tau+\tau_{p}\right)=Q(\tau), \quad P\left(\tau+\tau_{p}\right)=P(\tau),
$$

with one real period $\tau_{p}^{(1)}$ and one complex period $\tau_{p}^{(2)}$. This means that $\tau_{p}=n_{1} \tau_{p}^{(1)}+n_{2} \tau_{p}^{(2)}$, where $n_{1,2}=$ $0, \pm 1, \ldots$, and

$$
\begin{gathered}
\tau_{p}^{(1)}=2^{1 / 2}|g|^{-1 / 4} K\left(m_{J}\right), \\
\tau_{p}^{(2)}=i 2^{1 / 2}|g|^{-1 / 4} K^{\prime}\left(m_{J}\right) .
\end{gathered}
$$

Here $K\left(m_{J}\right)$ is the complete elliptic integral of the first kind, $K^{\prime}\left(m_{J}\right)=K\left(1-m_{J}\right)$, and $m_{J} \equiv m_{J}(g)$,

$$
m_{J}(g)=\frac{\left(\mu+1-2|g|^{1 / 2}\right)\left(\mu-1+2|g|^{1 / 2}\right)}{8|g|^{1 / 2}}-i 0
$$


is the parameter [38]. The real part of $m_{J}$ can be positive or negative; for $\operatorname{Re} m_{J}<0$, the period $\tau_{p}^{(2)}$ has not only imaginary, but also a nonzero real part.

The vibration frequency is $\omega(g)=2 \pi / \tau_{p}^{(1)} \equiv$ $2 \pi / \tau_{p}^{(1)}(g)$. It monotonically decreases with increasing $g$ in the range where $g(P, Q)$ has two wells, $-(\mu+1)^{2} / 4<$ $g<0$, and $\omega(g) \rightarrow 0$ for $g \rightarrow 0$, i.e. for $g$ approaching the saddle-point value. The periodicity of $Q(\tau), P(\tau)$ in imaginary time turns out to be instrumental in calculating matrix elements of the operators $P, Q$ on the intrawell wave functions $|n\rangle$ as discussed in the next section.

\section{The semiclassical matrix elements}

Of central interest to us will be the case where the number of states with $g_{n}<0$, that is the number of states inside the wells of $g(P, Q)$ in Fig. 4 is large. Formally, this case corresponds to the limit of a small effective Planck constant $\lambda$. For $\lambda \ll 1$ the wave function $|n\rangle$ in the classically accessible region can be written in the form

$$
\begin{array}{r}
|n\rangle=C^{\prime}\left(\partial_{P} g\right)^{-1 / 2} \exp \left[i S_{n}(Q) / \lambda\right]+\text { c.c. } \\
S_{n}(Q)=\int_{Q_{n}}^{Q} P\left(Q^{\prime}, g_{n}\right) d Q^{\prime} .
\end{array}
$$

Here, $C^{\prime}$ is the normalization constant, $P\left(Q, g_{n}\right)$ is the momentum for a given $Q$ as determined by the equation $g(P, Q)=g_{n}, Q_{n}$ is the classical turning point, $P\left(Q_{n}, g_{n}\right)=0$, and the derivative $\partial_{P} g$ is calculated for $P=P\left(Q, g_{n}\right)$.

We parameterize the classical trajectories (9) by their phase $\phi=\omega(g) \tau$, which we count (modulo $2 \pi$ ) from its value at $Q_{n}$. With this parametrization we have

$$
S_{n+m}(Q)-S_{n}(Q) \approx \lambda m \phi,
$$

for $|m| \ll \lambda^{-1}$; on a classical trajectory, $Q$ is an even and $P$ is an odd function of $\phi$.

With Eqs. (13) and (14), we can write the semiclassical matrix element of the lowering operator $\hat{a}=$ $(2 \lambda)^{-1 / 2}(P-i Q)$ as a Fourier component of $a(\phi ; g)=$ $(2 \lambda)^{-1 / 2}[P(\tau ; g)-i Q(\tau ; g)]$ over the phase $\phi=\omega(g) \tau$ [39],

$$
\begin{aligned}
\langle n+m|\hat{a}| n\rangle & \equiv a_{m}\left(g_{n}\right), \\
a_{m}(g) & =\frac{1}{2 \pi} \int_{0}^{2 \pi} d \phi \exp (-i m \phi) a(\phi ; g),
\end{aligned}
$$

to lowest order in $\lambda$. As we discuss below, these matrix elements determine relaxation of the oscillator.

We will be interested in calculating $a_{m}(g)$ for $g<0$. Then if we neglect interwell tunneling, we have two sets of wave functions $|n\rangle$, one for each well. The interwell matrix elements of the operator $\hat{a}$ are exponentially small. The intrawell matrix elements are the same for the both

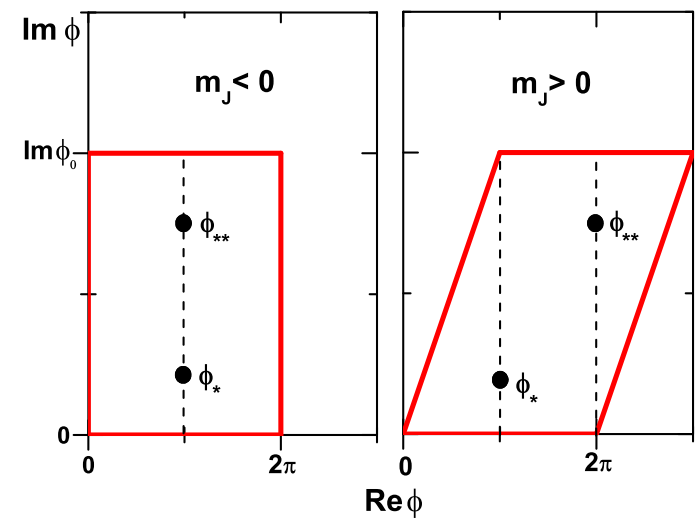

FIG. 5: (Color online) The contours of integration in the complex phase plane for calculating Fourier components of the oscillator coordinate $Q$ and momentum $P$. The left and right panels refer, respectively, to the negative and positive real part of the parameter of the elliptic functions $m_{J}$, Eq. (12) [the imaginary part of $m_{J}$ is infinitesimally small]. Both $Q$ and $P$ have poles at $\phi=\phi_{*}, \phi_{* *}$, but $a(\phi ; g)$ has a pole only for $\phi=\phi_{*}$. The increment of phase by $\phi_{0}$ leads to a transition between the trajectories with a given $g$ in different wells of $g(P, Q)$.

wells except for the overall sign, because $Q$ and $P$ in different wells have opposite signs on the trajectories with the same $g$. We will consider the matrix elements $a_{m}\left(g_{n}\right)$ for the states in the right well $Q>0$.

The integral (15) can be evaluated using the double periodicity of the functions $Q, P$. It is clear that $a(\phi+$ $2 \pi, g)=a(\phi ; g)$ for any complex $\phi$. In addition, from Eq. A3 for $g<0$ we have $a\left(\phi+\phi_{0}, g\right)=-a(\phi ; g)$, where $\phi_{0}=\pi\left(1+\tau_{p}^{(2)} / \tau_{p}^{(1)}\right)$ is a complex number, which is determined by the ratio of the periods of $Q$ and $P$. The shift in time by $\phi_{0} / \omega(g)$ corresponds to a transition from $Q(\tau), P(\tau)$ in one well to $Q(\tau), P(\tau)$ in the other well. We can now write the matrix element $a_{m}(g)$ as

$$
a_{m}(g)=\frac{1}{2 \pi}\left[1+e^{-i m \phi_{0}}\right]^{-1} \oint_{C} d \phi e^{-i m \phi} a(\phi ; g),
$$

where integration is done along the contour $C$ in Fig. 5 , It is explained in Appendix $\mathrm{A}$ that each of the functions $Q(\phi / \omega(g)), P(\phi / \omega(g))$ has two poles inside the contour $C$ [38]. Therefore it is easy to calculate the contour integral (16). The result has a simple form

$$
a_{m}(g)=-i(2 \lambda)^{-1 / 2} \omega(g) \frac{\exp \left(-i m \phi_{*}\right)}{1+\exp \left(-i m \phi_{0}\right)},
$$

with $\phi_{*}$ given by the equation

$$
\begin{aligned}
\operatorname{cn}\left(2 K \phi_{*} / \pi\right) & =-\left(\frac{1+\mu+2|g|^{1 / 2}}{1+\mu-2|g|^{1 / 2}}\right)^{1 / 2} \\
\operatorname{Im} \phi_{*} & <\frac{\pi}{2} \operatorname{Im}\left[\tau_{p}^{(2)} / \tau_{p}^{(1)}\right]=\frac{1}{2} \operatorname{Im} \phi_{0} .
\end{aligned}
$$


An important feature of the matrix elements $a_{m}(g)$ is their exponential decay for large $|m|$. From Eqs. (17) and (18)

$$
\begin{aligned}
a_{m}(g) \propto \exp \left[-m \operatorname{Im}\left(\phi_{0}-\phi_{*}\right)\right], & m \gg 1, \\
a_{m}(g) \propto \exp \left[-|m| \operatorname{Im} \phi_{*}\right], & -m \gg 1 .
\end{aligned}
$$

We note that the decay is asymetric with respect to the sign of $m$. This leads to important features of the probability distribution of the oscillator.

\section{BALANCE EQUATION}

Coupling of the oscillator to a thermal reservoir leads to its relaxation. We will first consider the simplest type of relaxation. It arises from coupling linear in the oscillator coordinate $q$ and corresponds to decay processes in which the oscillator makes transitions between neighboring energy levels, with energy $\approx \hbar \omega_{0}$ transferred to or absorbed from the reservoir. We will assume that the oscillator nonlinearity is not strong and that the detuning $\delta \omega$ of the modulation frequency is small, whereas the density of states of the reservoir weighted with interaction is smooth near $\omega_{0}$. Then the quantum kinetic equation for the oscillator density matrix $\rho$ in the rotating frame has the form

$$
\begin{array}{r}
\frac{\partial \rho}{\partial \tau}=i \lambda^{-1}[\rho, g]-\hat{\eta} \rho, \\
\hat{\eta} \rho=\eta\left[(\bar{n}+1)\left(\hat{a}^{\dagger} \hat{a} \rho-2 \hat{a} \rho \hat{a}^{\dagger}+\rho \hat{a}^{\dagger} \hat{a}\right)\right. \\
\left.+\bar{n}\left(\hat{a} \hat{a}^{\dagger} \rho-2 \hat{a}^{\dagger} \rho \hat{a}+\rho \hat{a} \hat{a}^{\dagger}\right)\right],
\end{array}
$$

where $\eta$ is the dimensionless relaxation constant and $\bar{n}=$ $\left[\exp \left(\hbar \omega_{0} / k T\right)-1\right]^{-1}$ is the Planck number.

We will assume that relaxation is slow so that the broadening of quasienergy levels is much smaller than the distance between them, $\eta \ll \omega(g)$. Then off-diagonal matrix elements of $\rho$ on the states $|n\rangle$ are small. We note that, at the same time, off-diagonal matrix elements of $\rho$ on the Fock states of the oscillator $|N\rangle$ do not have to be small.

To the lowest order in $\eta / \omega(g)$ relaxation of the diagonal matrix elements $\rho_{n}=\langle n|\rho| n\rangle$, is described by the balance equation

$$
\frac{\partial \rho_{n}}{\partial \tau}=-2 \eta \sum_{n^{\prime}}\left(W_{n n^{\prime}} \rho_{n}-W_{n^{\prime} n} \rho_{n^{\prime}}\right),
$$

with dimensionless transition probabilities

$$
W_{n n^{\prime}}=(\bar{n}+1)\left|\left\langle n^{\prime}|\hat{a}| n\right\rangle\right|^{2}+\bar{n}\left|\left\langle n|\hat{a}| n^{\prime}\right\rangle\right|^{2} .
$$

It follows from Eqs. (17) and (22) that, even for $T=0$, the oscillator can make transitions to states with both higher and lower $g$, with probabilities $W_{n n^{\prime}}$ with $n^{\prime}>n$ and $n^{\prime}<n$, respectively. This is in spite the fact that transitions between the Fock states of the oscillator for $T=0$ are only of the type $|N\rangle \rightarrow|N-1\rangle$.
The explicit expression for the matrix elements (17) makes it possible to show that the probability of a transition to a lower level of $\hat{g}$ is larger than the probability of a transition to a higher level, that is, $W_{n^{\prime} n}>W_{n n^{\prime}}$ for $n^{\prime}>n$. Therefore the oscillator is more likely to move down to the bottom of the initially occupied well in Fig. 4. This agrees with the classical limit in which the stable states of an underdamped parametric oscillator are at the minima of $g(P, Q)$. However, along with the drift down in the scaled period-two quasienergy $g$, even for $T=0$ there is also diffusion over quasienergy away from the minima of $g(P, Q)$, due to nonzero transition probabilities $W_{n n^{\prime}}$ with $n^{\prime}>n$.

Equation (21) must be slightly modified in the presence of tunneling between the states with $g_{n}<0$. The modification is standard. One has to take into account that the matrix elements of $\rho$ depend not only on the number $n$ of the period-two quasienergy level inside the well, but also on the index $\alpha$ which takes on two values $\alpha= \pm 1$, that specify the wells of $g(P, Q)$. These values can be associated with the eigenvalues of the pseudospin operator $\sigma_{z}$. The matrix elements of the operator $\hat{\eta} \rho$ on the wave functions of different wells are exponentially small and can be disregarded. The operator describing interwell tunneling can be written in the pseudospin representation as $-i(2 \lambda)^{-1} T(g)\left[\sigma_{x}, \rho\right]$, where $T(g)$ is the tunneling splitting of the states in different wells.

We assume that the tunneling splitting is much smaller than $\eta$. Then after a transient time $\tau \sim \eta^{-1}$, there is formed a quasistationary distribution over the states $|n\rangle$ inside each of the wells of $g(P, Q)$ in Fig. [4 This distribution can be found from Eq. (21) using the wave function $|n\rangle$ calculated in the neglect of tunneling. Interwell transitions occur over much longer time.

\section{DISTRIBUTION OVER INTRAWELL STATES}

The stationary intrawell probability distribution can be easily found from Eqs. (21) and (22) if the number of levels with $g_{n}<0$ is small. Much more interesting is the situation where this number is large. It corresponds to the limit of small $\lambda$. We will be interested primarily in the quasistationary distribution over the states in the well in which the system was initially prepared. It is formed over time $\sim \eta^{-1}$ and is determined by setting the right hand side of Eq. (21) equal to zero. The resulting equation describes also the stationary distribution in both wells, which is formed over a much longer time given by the reciprocal rate of interwell transitions.

For $\lambda \ll 1$ we can use the Wentzel-Kramers-Brillouin (WKB) approximation both to calculate the matrix elements in $W_{n n^{\prime}}$ (22) and to solve the balance equation. The solution should be sought in the eikonal form

$$
\rho_{n}=\exp \left[-R_{n} / \lambda\right], \quad R_{n}=R\left(g_{n}\right) .
$$

It follows from Eq. (19) that the transition probabilities 
$W_{n+m n}$ rapidly decay for large $|m|$. Therefore in the balance equation (21) we can set

$$
\begin{aligned}
& \rho_{n+m} \approx \rho_{n} \exp \left[-m \omega\left(g_{n}\right) R^{\prime}\left(g_{n}\right)\right], \\
& R^{\prime}(g)=d R / d g .
\end{aligned}
$$

The corrections to the exponent in Eq. (24) of order $\lambda m^{2} \omega^{2} R^{\prime \prime}, \lambda m^{2} \omega \omega^{\prime} R^{\prime}$ are small for $\lambda \ll 1$ (prime indicates differentiation over $\mathrm{g})$. We note that $\omega(g) R^{\prime}(g)$ is not small in the quantum regime and the exponential in (24) will not be expanded in a series in $R^{\prime}$.

From Eq. (21), the function $R^{\prime}(g)$ is determined by the polynomial equation

$$
\begin{aligned}
& \sum_{m} W_{n+m n}\left(1-\xi^{m}\right)=0, \\
& \xi=\exp \left[-\omega\left(g_{n}\right) R^{\prime}\left(g_{n}\right)\right] .
\end{aligned}
$$

Here the sum goes over positive and negative $m$. In obtaining Eq. (25) we have used the relation $W_{n+m n}=$ $W_{n n-m}$ for $|m| \ll n$, which is the consequence of the WKB approximation for the matrix elements: $\lambda \omega(g) d \ln \left[a_{m}(g)\right] / d g \ll 1$ for $\lambda \ll 1$.

Equation (25) has a trivial solution $\xi=1$, which is unphysical. Because all coefficients $W_{n+m n}$ are positive and $W_{n+m n}>W_{n-m n}$ for $m>0$, one can show that the polynomial in the left hand side of Eq. (25) has one extremum in the interval $0<\xi<1$. Since its derivative for $\xi=1$ is negative and it goes to $-\infty$ for $\xi \rightarrow 0$, it has one root in this interval. This root gives the value $R^{\prime}\left(g_{n}\right)$. Since $\xi<1$, we have $R^{\prime}(g)>0$. In turn $R^{\prime}(g)$ gives $R(g)$ and thus the distribution $\rho_{n}$. In obtaining $R(g)$ from $R^{\prime}(g)$, in the spirit of the eikonal approximation, one should set $R\left(g_{\min }\right)=0$, where $g_{\min }=-(\mu+1)^{2} / 4$ is the minimal quasienergy.

It is seen from Eq. (23) that $\lambda^{-1} R^{\prime}(g)$ has the meaning of the effective inverse temperature of the distribution

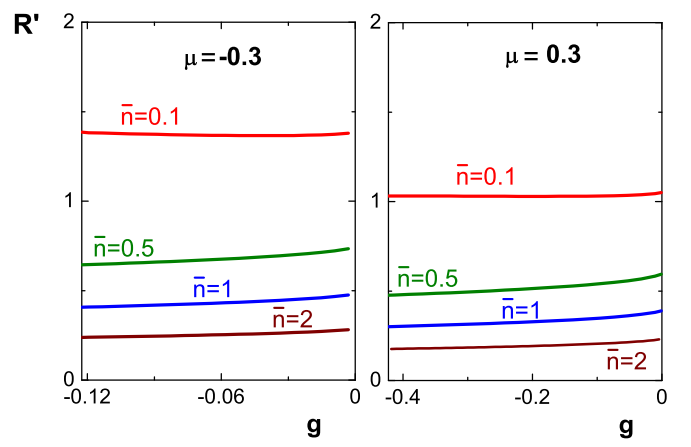

FIG. 6: (Color online) The scaled inverse temperature $R^{\prime}$ of the distribution over scaled period-two quasienergy $g$ for two values of the control parameter $\mu$ and for different oscillator Planck numbers $\bar{n}$. For $\bar{n} \gtrsim 0.1$ the function $R^{\prime}$ only weakly depends on $g$ inside the well, $g_{\min }<g<0$. over period-two quasienergy. The function $R^{\prime}(g)$ can be calculated for different values of the control parameter $\mu$ and different oscillator Planck numbers $\bar{n}$ by solving Eq. (25) numerically. The numerical calculation is simplified by the exponential decay of the coefficients $W_{n+m n}$ with $|m|$. The results are shown in Fig. 6] The function $R^{\prime}(g) / \lambda$ smoothly varies with $g$ in the whole range $g_{\min } \leq g<0$ of intrawell values of $g$, except for very small $\bar{n}$.

Numerical results on the logarithm of the distribution $R\left(g_{n}\right)=-\lambda \ln \rho_{n}$ obtained from Eq. (25) for $\bar{n}=0.1$ are compared in Fig 7(a) with the results of the full numerical solution of the balance equation (21). In this latter calculation we did not use the WKB approximation to find the transition probabilities $W_{n n^{\prime}}$. Instead they were obtained by solving numerically the Schrödinger equation $\hat{g}|n\rangle=g_{n}|n\rangle$ and by calculating $W_{n n^{\prime}}$ as the appropriately weighted matrix elements $\left|\left\langle n|\hat{a}| n^{\prime}\right\rangle\right|^{2}$, Eq. (22).

In the calculation we took into account that, for small $\lambda$, the levels of $\hat{g}$ form tunnel-split doublets. The tunneling splitting is small compared to the distance between intrawell states $\lambda \omega(g)$. The exact eigenfunctions of the operator $\hat{g}$ are well approximated by symmetric and antisymmetric combinations of the intrawell wave functions. This allows one to restore the intrawell wave functions from the full numerical solution and to calculate the matrix elements $W_{n n^{\prime}}$. By construction, such numerical approach gives $R(g)$ only for the values of $g$ that correspond to quasienergy levels $g_{n}$. It is seen from Fig. [7(a) that the two methods give extremely close results for small $\lambda$ (see also below).
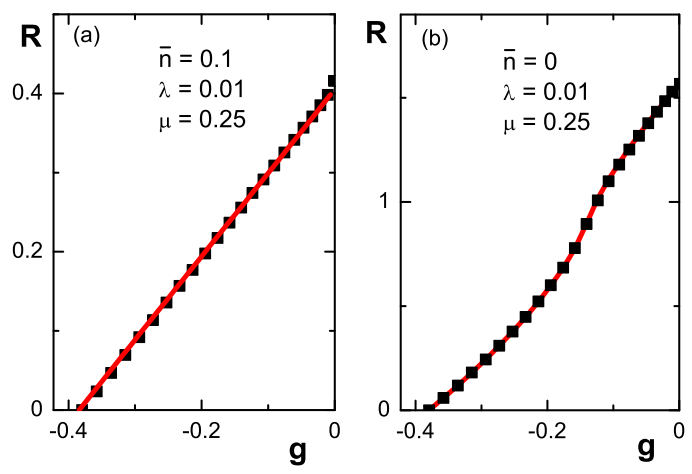

FIG. 7: (Color online) Comparison of the results of the eikonal approximation for the scaled logarithm of the probability distribution $R$ over period-two quasienergy $g$ (solid lines) with the results obtained by direct calculation of the transition probabilities followed by numerical solution of the balance equation (squares). 


\section{THE DISTRIBUTION IN LIMITING CASES}

The effective inverse temperature $R^{\prime}(g) / \lambda$ and the distribution $\rho_{n}$ can be found in several limiting cases. We start with the vicinity of the bottom of the wells of $g(P, Q)$. Here classical vibrations of $P, Q$ are nearly harmonic. To leading order in $\delta g=g-g_{\text {min }}$ we have $\langle n+m|\hat{a}| n\rangle \propto \delta_{|m|, 1} \delta g^{1 / 2}$ for $m \neq 0$, and therefore the transition probabilities $W_{n+m n} \propto \delta_{|m|, 1}$. Then Eq. (25) becomes a quadratic equation for $\xi$, giving

$$
\begin{aligned}
R^{\prime}\left(g_{\min }\right)= & \frac{1}{2}(\mu+1)^{-1 / 2} \\
& \times \ln \frac{(\mu+2)(2 \bar{n}+1)+2 \sqrt{\mu+1}}{(\mu+2)(2 \bar{n}+1)-2 \sqrt{\mu+1}} .
\end{aligned}
$$

The inverse effective temperature $R^{\prime}\left(g_{\min }\right)$ as given by Eq. (26) monotonically decreases with the increasing Planck number $\bar{n}$, i.e., with increasing bath temperature $T$. We note that $R^{\prime}\left(g_{\min }\right)$ smoothly varies with $\bar{n}$ for low temperatures $\bar{n} \ll 1$, except for small $|\mu|$. We have $\partial R^{\prime}\left(g_{\min }\right) / \partial \bar{n}=-2^{2 / 3}(\mu+1) / \mu$ for $\bar{n} \rightarrow 0$. For $\mu=0$, on the other hand, we have $R^{\prime}\left(g_{\min }\right) \approx \hbar \omega_{F} / 4 k T$, i.e. the effective inverse temperature for $g=g_{\min }$ is simply $\propto T^{-1}$.

We now consider the parameter ranges where $R^{\prime}(g)$ can be found for all $g$.

\section{A. Classical limit}

For $\bar{n} \gg 1$ the transition probabilities become nearly symmetric, $\left|W_{n+m n}-W_{n-m n}\right| \ll W_{n+m n}$. As a result, the effective inverse temperature $R^{\prime} / \lambda$ becomes small, and the ratio $\rho_{n+m} / \rho_{n}$ can be expanded in $R^{\prime}\left(g_{n}\right) / \lambda$. This gives

$$
R^{\prime}(g)=2 \omega^{-1}(g) \sum_{m} m W_{n+m n} / \sum_{m} m^{2} W_{n+m n} .
$$

It is shown in Appendix B that Eq. (27) can be written in the simple form

$$
\begin{array}{r}
R^{\prime}(g)=\frac{2}{2 \bar{n}+1} M(g) / N(g), \\
M(g)=\iint_{A(g)} d Q d P, \\
N(g)=\frac{1}{2} \iint_{A(g)} d Q d P\left(\partial_{Q}^{2} g+\partial_{P}^{2} g\right),
\end{array}
$$

where the integration is performed over the area $A(g)$ of the phase plane $(\mathrm{Q}, \mathrm{P})$ encircled by the classical trajectory $Q(\tau), P(\tau)$ with given $g$.

From Eq. (28), the effective inverse temperature $R^{\prime} / \lambda$ is $\propto[\lambda(2 \bar{n}+1)]^{-1}$. In the high-temperature limit $(2 \bar{n}+$ $1) \approx 2 k T / \hbar \omega_{0}$. Therefore $R^{\prime} / \lambda$ is $\propto T^{-1}$ and does not contain $\hbar$, as expected. Eq. (28) in this limit coincides with the expression for the distribution obtained in Ref. 37 using a completely different method.

\section{B. Vicinity of the bifurcation point}

The function $R^{\prime}(g)$ may be expected to have a simple form for $\mu$ close to the bifurcation value $\mu_{B}=-1$ where the two stable states of the oscillator merge together and $g(P, Q)$ becomes single well. This is a consequence of the universality that characterizes the dynamics near bifurcation points and is related to the slowing down of motion and the onset of a soft mode. The situation we are considering here is not the standard situation of the classical theory where the problem is reduced to fluctuations of the soft mode. The oscillator is not too close to the bifurcation point, its motion is not overdamped and the interlevel distance exceeds the level broadening. Still as we show $R(g)$ has a simple form.

The motion slowing down for $\mu$ approaching -1 leads to the decrease of the vibration frequencies. From Eq. (11),

$$
\omega(g)=2 \pi / \tau_{p}^{(1)} \propto|g|^{1 / 4} \leq[(\mu+1) / 2]^{1 / 2}
$$

for $\mu+1 \ll 1$. Therefore one may expect that $\omega(g) R^{\prime}(g)$ becomes small, and we can again expand $\rho_{n+m} / \rho_{n}$ in $\omega\left(g_{n}\right) R^{\prime}\left(g_{n}\right)$, as in Eq. (27). One can justify this expansion more formally by noticing that the transition probabilities $W_{n+m n}$ are nearly symmetric near the bifurcation point $\left|\left(W_{n+m n}-W_{n-m n}\right)\right| \ll W_{n-m n}$. In the present case the latter inequality is a consequence of the relation $\operatorname{Im}\left(\phi_{0}-2 \phi_{*}\right) \ll 1$ in Eq. (17), which leads to ||$a_{-m}(g)|-| a_{m}(g)|| \ll\left|a_{m}(g)\right|$. In turn, the above relation between $\phi_{0}$ and $\phi_{*}$ can be obtained from Eqs. (12) and (18), which show that the right-hand side of Eq. (18) is $\approx-\left|m_{J} /\left(1-m_{J}\right)\right|^{1 / 2}$ for $\mu+1 \ll 1$. Since $\left|\operatorname{cn}\left(K+i K^{\prime}\right)\right|=\left|m_{J} /\left(1-m_{J}\right)\right|^{1 / 2}$ [38], we have from Eqs. (11) and (18) $\operatorname{Im} \phi_{*} \approx \operatorname{Im} \tau_{p}^{(2)} / 2 \tau_{p}^{(1)}=\operatorname{Im} \phi_{0} / 2$.

It follows from the above arguments that near the bifurcation point $R^{\prime}(g)$ is given by Eq. (28) for arbitrary Planck number $\bar{n}$, i.e., for arbitrary temperature. Since $\partial_{Q}^{2} g+\partial_{P}^{2} g \approx 2$ for $\mu+1 \ll 1$, we obtain from Eq. (28) a simple explicit expression

$$
R^{\prime}(g) \approx 2 /(2 \bar{n}+1),
$$

Eq. (29) agrees with Eq. (26) near $g_{\min }$ in the limit $\mu+$ $1 \ll 1$. It shows that the inverse effective temperature $R^{\prime} / \lambda$ is independent of $g$. It monotonically decreases with increasing temperature $T$.

\section{Zero temperature: detailed balance}

The function $R^{\prime}(g)$ can be also obtained for $T=\bar{n}=0$. This is a consequence of detailed balance that emerges in this case [34]. The detailed balance condition is usually a consequence of time reversibility, which does not characterize the dynamics of a periodically modulated oscillator. So, in the present case detailed balance comes from a special relation between the parameters for $T=0$. 
Detailed balance means that transitions back and forth between any two states are balanced. It is met if the ratio of the probabilities of direct transitions between two states is equal to the ratio of probabilities of transitions via an intermediate state

$$
\frac{W_{n n^{\prime}}}{W_{n^{\prime} n}}=\frac{W_{n n^{\prime \prime}} W_{n^{\prime \prime} n^{\prime}}}{W_{n^{\prime} n^{\prime \prime}} W_{n^{\prime \prime} n}} .
$$

One can see from Eqs. (17) and (22) that the condition (30) is indeed met for $\bar{n}=0$. Therefore the balance equation has a solution $\rho_{n} / \rho_{n^{\prime}}=W_{n^{\prime} n} / W_{n n^{\prime}}$, which immediately gives

$$
R^{\prime}(g)=2 \omega^{-1}(g) \operatorname{Im}\left(\phi_{0}-2 \phi_{*}\right) .
$$

The function $R^{\prime}(g)$ is plotted in Fig. 8 for two values of the control parameter $\mu$. It displays different behavior depending on the sign of $\mu$. For $\mu<0$ the parameter of the elliptic function $m_{J}$, Eq. (12), is negative for all $g<0$. Therefore the periods $\tau_{p}^{(1,2)}(g)$ of $P(\tau), Q(\tau)$, Eq. (11), are smooth functions of $g$, except near $g_{\min }=$ $-\frac{1}{4}(\mu+1)^{2}$, where $\operatorname{Im} \tau_{p}^{(2)} \approx(1+\mu)^{-1 / 2}\left|\ln \left(g-g_{\min }\right)\right|$. This divergence of $\operatorname{Im} \tau_{p}^{(2)}$ is seen in Fig. 8. The function $R^{\prime}(g)$ remains finite near $g_{\min }$, Eq. (26).
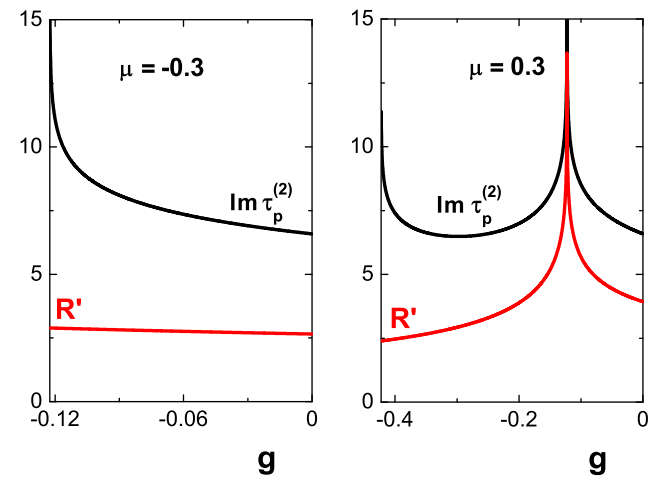

FIG. 8: (Color online) The scaled inverse temperature $R^{\prime}$ and the imaginary part of the period $\tau_{p}^{(2)}$ of the oscillator coordinate and momentum $Q(\tau ; g), P(\tau ; g)$ for $T=0$. For $\mu>0$ both $R^{\prime}$ and $\operatorname{Im} \tau_{p}^{(2)}$ have a logarithmic singularity.

For $\mu>0$, on the other hand, the function $R^{\prime}(g)$ has a singularity. Its location $g_{d}$ is determined by the condition $m_{J}=0$, which gives $g_{d}=-(1-\mu)^{2} / 4$. For small $\mid g-$ $g_{d} \mid$ we have $\operatorname{Im} \phi_{0} \propto \operatorname{Im} \tau_{p}^{(2)} \propto\left|\ln \left(\left|g-g_{d}\right|\right)\right|$, whereas $\phi_{*}$, Eq. (18) remains finite for $g=g_{d}$. Therefore $R^{\prime}(g)$ diverges logarithmically at $g_{d}$, as seen from Fig. 8 (b).

Physically the divergence of the inverse temperature is related to the structure of the transition probabilities $W_{n n^{\prime}}$ for $\bar{n}=0$. For $g \rightarrow g_{d}$ we have $W_{n n+m} \propto$ $\left(g_{n}-g_{d}\right)^{2 m}$ for $m>0$, see Eqs. (17) and (22). This means that, for $g_{n}$ close to $g_{d}$, diffusion towards larger $g$ slows down. The slowing down leads to a logarithmic singularity of $R^{\prime}(g)$. The eikonal approximation is inapplicable for $g$ close to $g_{d}$. However, the width of the range of $g$ where this happens is small, $\delta g \sim \lambda$, as follows from the discussion below Eq. (24). In addition, there are corrections to the balance equation due to off-diagonal terms in the full kinetic equation. These corrections give extra terms $\propto \eta^{2} / \omega^{2}\left(g_{d}\right)$ in the transition probabilities $W_{n n^{\prime}}$. The analysis of these corrections as well as features of $R$ that are not described by the eikonal approximation is beyond the scope of this paper because, as we show, these features are fragile.

The function $R\left(g_{n}\right)=-\lambda \ln \rho_{n}$ obtained by integrating Eq. (31) is compared in Fig. 7(b) with the result of the numerical solution of the balance equation (21) with numerically calculated transition probabilities $W_{n n^{\prime}}$. The semiclassical and numerical results are in excellent agreement. We checked that the agreement persists for different values of the control parameter $\mu$ and for almost all $\lambda \ll 1$, except for a few extremely narrow resonant bands of $\lambda$.

\section{SWITCHING EXPONENT}

Quantum diffusion over quasienergy described by Eq. (21) leads to switching between the classically stable states of the oscillator at the minima of $g(P, Q)$ in Fig. 4 . The switching rate $W_{\mathrm{sw}}$ is determined by the probability to reach the top of the barrier of $g(P, Q)$, that is by the distribution $\rho_{n}$ for such $n$ that $g_{n}=0$. To logarithmic accuracy

$$
W_{\mathrm{sw}}=C_{\mathrm{sw}} \times \exp \left(-R_{A} / \lambda\right), \quad R_{A}=\int_{g_{\mathrm{min}}}^{0} R^{\prime}(g) d g,
$$

where $R^{\prime}(g)$ is given by Eq. (25). The parameter $C_{\mathrm{sw}}$ is of the order of the relaxation rate $\eta$ due to coupling to a thermal bath.

The quantity $R_{A}$ plays the role of the activation energy of escape. The activation is due to quantum fluctuations that accompany relaxation of the oscillator, and we call it quantum activation energy. As we show in Sec. VIII $R_{A}$ is smaller than the tunneling exponent for tunneling between the minima of $g(P, Q)$. Therefore if the relaxation rate $\eta$ exceeds the tunneling rate, switching between the stable states occurs via quantum activation.

Quantum activation energy $R_{A}$ obtained by solving Eq. 25) numerically for $R^{\prime}$ is plotted in Fig. 3. It depends on the control parameter $\mu$ and the Planck number $\bar{n}$, and it monotonically increases with increasing $\mu$ and decreasing $\bar{n}$. Close to the bifurcation point $\mu_{B}=-1$, i.e., for $\mu-\mu_{B} \ll 1$, it displays scaling behavior with $\mu-\mu_{B}$. From Eq. (29)

$$
R_{A}=\frac{1}{2}(2 \bar{n}+1)^{-1}\left(\mu-\mu_{B}\right)^{\zeta}, \quad \zeta=2 .
$$

The scaling exponent $\zeta=2$ coincides with the scaling exponent near the bifurcation point of a classical para- 


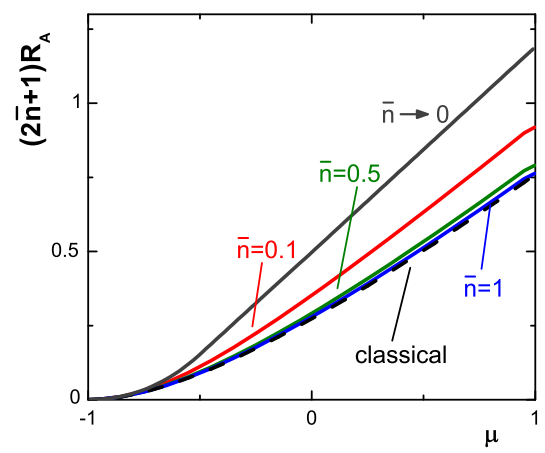

FIG. 9: (Color online) Quantum activation energy of switching between the states of parametrically excited vibrations for different oscillator Planck numbers $\bar{n}$ as a function of the scaled frequency detuning $\mu$. The transition rate is $W_{\text {sw }} \propto \exp \left(-R_{A} / \lambda\right)$. With increasing $\bar{n}$, the value of $R_{A}$ multiplied by $2 \bar{n}+1$ quickly approaches the classical limit $\bar{n} \gg 1$ shown by the dashed line. In this limit the ratio $R_{A} / \lambda$ is $\propto T^{-1}$ and does not contain $\hbar$.

metric oscillator where the oscillator motion is still underdamped in the rotating frame, i.e., $\mu-\mu_{B}$ is not to small [37]. It can be seen from the results of Refs. 40, 41 that the exponent $\zeta=2$ also describes scaling of the activation energy of escape due to classical fluctuations closer to the pitchfork bifurcation point where the motion is necessarily overdamped.

In the classical limit $2 \bar{n}+1 \gg 1$ we have from Eq. (28) $R_{A} \propto(2 \bar{n}+1) \propto T^{-1}$, i.e., the switching rate $W_{\mathrm{sw}} \propto$ $\exp \left[-\left(R_{A} k_{B} T / \lambda\right) / k_{B} T\right]$, with temperature independent $R_{A} k_{B} T / \lambda$ being the standard activation energy. The quantity $R_{A}(2 \bar{n}+1)$ in the classical limit as obtained from Eq. (28), is shown with the dashed line in Fig. 9 , It is seen that $R_{A}$ quickly approaches the classical limit with increasing Planck number $\bar{n}$, so that even for $\bar{n}=0.1$ the difference between $R_{A}(2 \bar{n}+1)$ and its classical limit is $\lesssim 15 \%$. Effectively it means that, for small $\bar{n} \ll 1$, the exponent in the probability of quantum activation can be approximated by the classical exponent for activated switching in which one should replace

$$
k_{B} T \rightarrow \hbar \omega_{0} .
$$

\section{FRAGILITY OF THE DETAILED BALANCE SOLUTION}

It turns out that the expression for the distribution (23) and (31) found from the detailed balance condition for $T=0$ does not generally apply even for infinitesimally small but nonzero temperature, in the semiclassical limit. This is a consequence of this solution being of singular nature, in some sense. A periodically modulated oscillator should not have detailed balance, because the underlying time reversibility is broken; the detailed balance condition (30) is satisfied just for one value of $\bar{n}$ and when other relaxation mechanisms are disregarded.

Formally, in a broad range of $\mu$ the correction $\propto \bar{n}$ to the $T=0$ solution diverges. The divergence can be seen from Eqs. (19), (22), (31). The transition probabilities $W_{n n+m}$ have terms $\propto \bar{n}$ which vary with $m$ as $\exp \left[-2 m \operatorname{Im} \phi_{*}\right]$ for $m \gg 1$. At the same time, the $T=0$ solution (31) gives $\xi^{-m}=\exp \left[m \omega(g) R^{\prime}(g)\right]=$ $\exp \left[2 m \operatorname{Im}\left(\phi_{0}-2 \phi_{*}\right)\right]$. Therefore for the series (25) with the term $\propto \bar{n}$ in $W_{n n+m}$ to converge we have to have

$$
\operatorname{Im}\left(\phi_{0}-3 \phi_{*}\right)<0 .
$$

The condition (35) is met at the bottom of the wells of $g(P, Q)$ and also close to the bifurcation values of the control parameter, $\mu-\mu_{B} \ll 1$. We found that, with increasing $\mu$, the condition (35) is broken first for $g$ approaching the barrier top, $g \rightarrow 0$. In this region $\operatorname{Im} \phi_{0}, \operatorname{Im} \phi_{*} \propto|\ln | g||^{-1}$. A somewhat tedious calculation based on the properties of the elliptic functions shows that the condition (35) is violated when $\mu>-1 / 2$, and for $\mu=-1 / 2$ we have $\operatorname{Im}\left(\phi_{0}-3 \phi_{*}\right) \rightarrow 0$ for $g \rightarrow 0$. The increase in $\mu$ leads to an increase of the range of $g$ where Eq. (35) does not apply. The detailed balance distribution is inapplicable for $T \rightarrow 0$ in this range.

To find the distribution for small Planck number $\bar{n}$ in the range where $\operatorname{Im}\left(\phi_{0}-3 \phi_{*}\right)>0$ we seek the solution of the balance equation (25) in the form

$$
R^{\prime}(g)=2 \omega^{-1}(g)\left[\operatorname{Im} \phi_{*}(g)-\epsilon\right], \epsilon \ll 1 .
$$

This solution does not give diverging terms for $\bar{n}>0$. The terms $W_{n n+m} \exp \left[m \omega(g) R^{\prime}(g)\right]$ in Eq. (25) are $\propto$ $\bar{n} \exp (-2 m \epsilon)$ for $m \gg 1$, and their sum over $m$ is $\propto \bar{n} / \epsilon$. Using the explicit expression for the transition probabilities we obtain that, to leading order in $\bar{n}$ 


$$
\epsilon=\bar{n}\left[\sum_{m} \exp \left[m \operatorname{Im}\left(\phi_{0}-3 \phi_{*}\right)\right] \sinh \left(m \operatorname{Im} \phi_{*}\right) /\left|\cos \left(m \operatorname{Im} \phi_{0} / 2\right)\right|^{2}\right]^{-1}
$$

Here again, the sum goes over positive and negative $m$. In obtaining Eq. (37) we used the general expression (17) for the coefficients $a_{m}(g)$ in $W_{n+m n}$. In fact, the asymptotic expressions (19) for $a_{m}(g)$ allow one to calculate the sum over $m$ in Eq. (37) explicitly and the result provides a very good approximation for $\epsilon$.

Equations (36) and (37) give the effective inverse temperature $R^{\prime} / \lambda$ and therefore the distribution $\rho_{n}$ as a whole for $\bar{n} \ll 1$. It is clear that the $\bar{n} \rightarrow 0$ limit is completely different from the detailed balance solution (31) for $\bar{n}=0$, that is, the transition to the $T=\bar{n}=0$ regime is nonanalytic. The difference between the $\bar{n}=0$ and the $\bar{n} \rightarrow 0$ solution is seen in Figs. 2 and 3 .

It follows from Eq. (37) that the perturbation theory diverges for the value of $g$ where $\operatorname{Im}\left(\phi_{0}-3 \phi_{*}\right)=0$. At such $g$ the $\bar{n} \rightarrow 0$ solution (36) coincides with the $\bar{n}=0$ solution (31). For smaller $g$ the condition (35) is satisfied and the $T=0$ solution for $R^{\prime}(g)$ applies; the corrections to this solution are $\propto \bar{n} \ll 1$. The derivative of the effective inverse temperature $R^{\prime}(g) / \lambda$ over $g$ is discontinuous at the crossover between $\bar{n} \rightarrow 0$ and $\bar{n}=0$ solutions, as seen in Fig. 2. Figure 2 illustrates also the smearing of the singularity of $R^{\prime}(g)$ due to terms $\propto \bar{n}$ in $W_{n n^{\prime}}$, which is described by the numerical solution of the balance equation (25). Away from the crossover the analytical solutions provide a good approximation to numerical results.

\section{A. Breaking of detailed balance solution by dephasing}

Dephasing plays an important role in the dynamics of quantum systems. It comes from fluctuations of the transition frequency due to external noise or to coupling to a thermal reservoir. A simple mechanism is quasielastic scattering of excitations of the reservoir off the quantum system. Since the scattering amplitude depends on the state of the system, the scattering leads to diffusion of the phase difference of different states.

For an oscillator, dephasing has been carefully studied, both microscopically and phenomenologically, see Refs. 42, 43, 44 and papers cited therein. It leads to an extra term $-\hat{\eta}^{\mathrm{ph}} \rho$ in the quantum kinetic equation (20), with

$$
\hat{\eta}^{\mathrm{ph}} \rho=\eta^{\mathrm{ph}}\left[\hat{a}^{\dagger} \hat{a},\left[\hat{a}^{\dagger} \hat{a}, \rho\right]\right]
$$

where $\eta^{\mathrm{ph}}$ is the dimensionless dephasing rate.

If both $\eta_{\mathrm{ph}}$ and $\eta$ are small compared to $\omega(g)$, populations of the steady states $\rho_{n}$ are described by the balance equation (21) in which one should replace $\eta W_{n^{\prime} n} \rightarrow$

$$
\begin{aligned}
& \eta W_{n^{\prime} n}+\eta^{\mathrm{ph}} W_{n^{\prime} n}^{(\mathrm{ph})}, \text { with } \\
& \qquad W_{n^{\prime} n}^{(\mathrm{ph})}=\left|\left\langle n\left|\hat{a}^{\dagger} \hat{a}\right| n^{\prime}\right\rangle\right|^{2} .
\end{aligned}
$$

It follows from Eq. (19) that, for large $\left|n^{\prime}-n\right|$, we have $W_{n^{\prime} n}^{(\mathrm{ph})} \propto \exp \left[-2\left|n^{\prime}-n\right| \operatorname{Im} \phi_{*}\right]$, that is, the transition probability exponentially decays with increasing $\left|n^{\prime}-n\right|$, and the exponent is determined by $\operatorname{Im} \phi_{*}$.

Even slow dephasing is sufficient for making the detailed balance condition inapplicable. Mathematically, the effect of slow dephasing is similar to the effect of nonzero temperature. If the condition (35) is violated, the sum $\sum W_{n+m n}^{(\mathrm{ph})} \exp \left(-m \omega\left(g_{n}\right) R^{\prime}\left(g_{n}\right)\right)$ with $R^{\prime}(g)$ given by the detailed balance solution (31) diverges. The correct distribution for the appropriate $g$ and $\mu$ is given by Eq. (36). The parameter $\epsilon \equiv \epsilon(g)$ is given by Eq. (37) in which $\bar{n}$ is replaced,

$$
\begin{aligned}
& \bar{n} \rightarrow \bar{n}+C^{\mathrm{ph}} \eta^{\mathrm{ph}} / \eta, \quad C^{\mathrm{ph}}=\omega^{2}(g) / 2 \lambda \\
& \times\left|\sum_{m=0}^{\infty} \exp \left(2 i m \phi_{*}\right) /\left[\exp \left(i m \phi_{0}\right)+1\right]\right|^{2} .
\end{aligned}
$$

It is seen from Eq. (40) and Figs. 2 and 3 that, for low temperatures, even weak dephasing, $\eta^{\mathrm{ph}} / \eta \ll 1$, leads to a very strong change of the probability distribution.

The fragility of the detailed balance solution discussed in this section is a semiclassical effect. It occurs if the number of states in each well $N \propto \lambda^{-1}$ is large. Formally we need $\epsilon \exp \left[2 c N \operatorname{Im}\left(\phi_{0}-3 \phi_{*}\right)\right] \gtrsim 1$, with $c=c(g) \sim 1$. In other words, the detailed balance solution is fragile provided $\lambda$ is sufficiently small. A full numerical solution of the balance equation confirms that, when $\lambda$ is no longer small, the distribution for small $\bar{n}, \eta^{\mathrm{ph}} / \eta$ is close to that described by the $\bar{n}=0, \eta^{\mathrm{ph}} / \eta=0$.

\section{TUNNELING}

The oscillator localized initially in one of the wells of the effective Hamiltonian $g(P, Q)$ can switch to another well via tunneling. For small $\lambda$ tunneling can be described in the WKB approximation. We will first find the tunneling exponent assuming that the oscillator is in the lowest intrawell state and show that it exceeds the quantum activation exponent $R_{A} / \lambda$. We will then use standard arguments to show that this is true independent of the initially occupied intrawell state, for all temperatures.

If we now go back to the original problem of switching between stable states of a periodically modulated oscillator, we see that switching via tunneling can be observed 
only where the relaxation rate is smaller than the tunneling rate, that is the prefactor in the rate of quantum activation is very small. Such experiment requires preparing the system in one of the wells. Tunneling between period two states of a strongly modulated strongly nonlinear system has been demonstrated in atomic optics [45, 46, 47]. For a weakly nonlinear oscillator, tunneling splitting between the lowest states of the Hamiltonian $\hat{g}$ for $\mu=0$ was found in Ref. [48].

The effective Hamiltonian $g(P, Q)$ is quartic in the momentum $P$. Therefore the semiclassical momentum $P(Q, g)$ as given by the equation $g(P, Q)=g$ has 4 rather than 2 branches. This leads to new features of tunneling compared to the standard picture for one-dimensional systems with a time-independent Hamiltonian quadratic in $P$.

For concreteness, we will consider tunneling from the left well, which is located at $Q=Q_{l 0}=-(1+\mu)^{1 / 2}, P=$ 0 . The rate of tunneling from the bottom of the well of $g(P, Q)$ in Fig. 4 is determined by the WKB wave function with $g=g_{\min }=-(1+\mu)^{2} / 4$. This wave function is particularly simple for $\mu<0$. In the region $Q_{l 0}<Q$ and not too close to $Q_{l 0}$ it has the form

$$
\begin{aligned}
& \left|\psi_{l}\right\rangle=C\left(\partial_{P} g\right)^{-1 / 2} \exp \left[i S_{0}(Q) / \lambda\right], \\
& S_{0}(Q)=\int_{Q_{l 0}}^{Q} P_{-}\left(Q^{\prime}\right) d Q^{\prime},
\end{aligned}
$$

where

$$
P_{ \pm}(Q)=i\left[1 \pm\left(Q^{2}-\mu\right)^{1 / 2}\right] .
$$

For $\mu<0$ the wave function $\left|\psi_{l}\right\rangle$ monotonically decays with increasing $Q$. The exponent for interwell tunneling is $S_{\text {tun }} / \lambda$, with $S_{\text {tun }}=\operatorname{Im} S_{0}\left(-Q_{l 0}\right)$ (we use that $-Q_{l 0}$ is the position of the right well),

$$
S_{\text {tun }}=(1+\mu)^{1 / 2}+\mu \log \frac{1+(1+\mu)^{1 / 2}}{|\mu|^{1 / 2}} .
$$

A more interesting situation arises in the case $\mu>$ 0 . Here, inside the classically forbidden region $-\mu^{1 / 2}<$ $Q<\mu^{1 / 2}$ decay of the wave function is accompanied by oscillations. We leave the analysis of this behavior for a separate paper. Here we only note that the tunneling exponent is still given by Eq. (43).

If the level splitting due to tunneling is small compared to their broadening due to relaxation, the tunneling probability is quadratic in the tunneling amplitude,

$$
W_{\text {tun }} \propto \exp \left(-2 S_{\text {tun }} / \lambda\right) .
$$

The action $2 S_{\text {tun }}$ as a function of $\mu$ is plotted in Fig. 10. It is seen from this figure that the tunneling exponent exceeds the quantum activation exponent $R_{A} / \lambda$ for all values of the control parameter $\mu$. This indicates that, as mentioned above, it is exponentially more probable to switch between the classically stable states of the oscillator via activation than via tunneling from the ground intrawell state, for not too small relaxation rate.

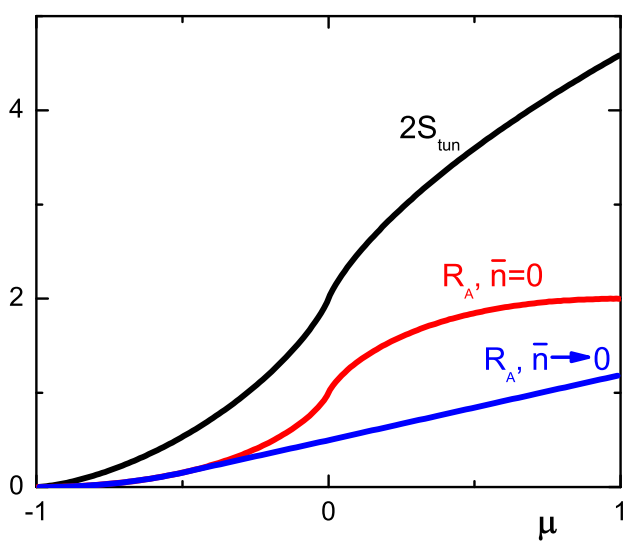

FIG. 10: (Color online) The scaled exponent $2 S_{\text {tun }}$ in the tunneling probability as a function of the parameter $\mu=$ $2 \omega_{F} \delta \omega / F$ (solid line). Also shown for comparison is the quantum activation energy $R_{A}$ for $\bar{n}=0$ and $\bar{n} \rightarrow 0$.

In the same limit where the relaxation rate exceeds the tunneling rate, we can consider the effect of tunneling from excited intrawell states of the Hamiltonian $\hat{g}$. The analysis is similar to that for systems in thermal equilibrium [1, 2, 3]. Over the relaxation time there is formed a quasiequilibrium distribution over the states inside the initially occupied well of $g(P, Q)$. As before, we assume that relaxational broadening of the levels $g_{n}$ is small compared to the level spacing. The probability of tunneling from a given state $n$ is determined by its occupation $\rho_{n}$. The overall switching probability is given by a sum of tunneling probabilities from individual intrawell states

$$
W_{\mathrm{sw}}=\sum_{n} C_{n} e^{-2 S_{n} / \lambda} \rho_{n},
$$

where $C_{n}$ is a prefactor that smoothly depends on $n$, and

$$
S_{n}=\operatorname{Im} \int P\left(Q, g_{n}\right) d Q
$$

is the imaginary part of the action of a classical particle with Hamiltonian $g(P, Q)$ and energy $g_{n}$, which moves in complex time from the turning point $P=0$ in one well of $g(P, Q)$ to the turning point in the other well. It follows from the analysis in Appendix A and Eq. (A3), that the duration of such motion is $\left(\tau_{p}^{(1)}+\tau_{p}^{(2)}\right) / 2$. Therefore $\partial S_{n} / \partial g_{n}=-\operatorname{Im} \tau_{p}^{(2)} / 2$.

Taking into account that $\rho_{n}=\exp \left(-R_{n} / \lambda\right)$, we see that the derivative of the overall exponent in Eq. (45) over $g_{n}$ is $\operatorname{Im} \tau_{p}^{(2)}-R^{\prime}\left(g_{n}\right)$. It was shown in Secs. IV andV that this derivative is always positive, cf. Fig. 8 . Therefore the exponent monotonically increases (decreases in absolute value) with increasing $g$. This shows that, with 
overwhelming probability, switching occurs via overbarrier transitions, i.e., the switching mechanism is quantum activation. We emphasize that this result is independent of the bath temperature.

\section{CONCLUSIONS}

In this paper we studied switching between the states of period two vibrations of a parametrically modulated nonlinear oscillator and the distribution over period-two quasienergy levels. We considered a semiclassical case where the wells of the scaled oscillator Hamiltonian in the rotating frame $g(P, Q)$, Fig. 4, contain many levels. The distance between the levels is small compared to $\hbar \omega_{F}$ but much larger than the tunneling splitting. We assumed that the oscillator is underdamped, so that the interlevel distance exceeds their width. At the same time, of primary interest was the case where this width exceeds the tunneling splitting.

We considered relaxation due to coupling to a thermal bath, which is linear in the oscillator coordinate, as well as dephasing from random noise that modulates the oscillator frequency. The problem of the distribution over intrawell states was reduced to a balance equation. The coefficients in this equation were obtained explicitly in the WKB approximation, using the analytical properties of the solution of the classical equations of motion. The balance equation was then solved in the eikonal approximation. The eikonal solution was confirmed by a full numerical solution of the balance equation, which did not use the WKB approximation for transition matrix elements.

We found that the distribution over period-two quasienergy has a form of the Boltzmann distribution with effective temperature that depends on the quasienergy. This temperature remains nonzero even where the temperature of the thermal bath $T \rightarrow 0$. It is determined by diffusion over quasienergy, which accompanies relaxation and has quantum origin: it is due to the Floquet wave functions being combinations of the Fock wave functions of the oscillator.

Unexpectedly, we found that the quasienergy distribution for $T=0$, where the system has detailed balance, is fragile. It differs significantly from the solution for $T \rightarrow 0$, for a large number of intrawell states. The $T=0$ solution is also destroyed by even small dephasing.

The probability of switching between period two states $W_{\mathrm{sw}}$ is determined by the occupation of the states near the barrier top of the effective Hamiltonian $g(P, Q)$. We calculated the effective quantum activation energy $R_{A}$ which gives $W_{\mathrm{sw}} \propto \exp \left(-R_{A} / \lambda\right)$. Both $R_{A} / \lambda$ and the exponent of the tunneling probability are proportional to the reciprocal scaled Planck constant $\lambda^{-1}$. However, for all parameter values and all bath temperatures, $R_{A} / \lambda$ is smaller than the tunneling exponent. Therefore in the case where intrawell relaxation is faster than interwell tunneling, switching occurs via quantum activation.
In the limit where fluctuations of the oscillator are classical, $k T \gg \hbar \omega_{F}$, we have $R_{A} \propto(k T)^{-1}$, and $W_{\text {sw }}$ is described by the standard activation law. Down to small Planck numbers $\bar{n} \gtrsim 0.1$ the quantum activation energy $R_{A}$ is reasonably well described by the classical expression even for small $k T / \hbar \omega_{F}$ provided $k T$ is replaced by $\hbar \omega_{F}(2 \bar{n}+1) / 4$, with $\bar{n}$ being the Planck number of the oscillator. The inapplicability of this description for small $\bar{n}$ indicates, however, that classical and quantum fluctuations do not simply add up. The replacement $k T \rightarrow \hbar \omega_{F}(2 \bar{n}+1) / 4$ becomes exact for all $\bar{n}$ close to the bifurcational value of the control parameter $\mu=\mu_{B}$ where the period-two states first emerge. In this range $R_{A}$ scales with the distance to the bifurcation point as $R_{A} \propto\left(\mu-\mu_{B}\right)^{2}$.

The results on switching rate are accessible to direct experimental studies in currently studied nano- and microsystems, in particular in systems based on Josephson junctions, including those used for highly sensitive quantum measurements.

We gratefully acknowledge a discussion with M. Devoret. This work was partly supported by the NSF through grant No. ITR-0085922.

\section{APPENDIX A: CLASSICAL MOTION OF THE PARAMETRIC OSCILLATOR}

The Hamiltonian $g(P, Q)$ (6) is quartic in $P$ and $Q$. This makes it possible to solve classical equations of motion (9). Trajectories with given $g$ lie on the cross-section $g(P, Q)=g$ of the surface $g(P, Q)$ in Fig. 4. We will be interested only in the intrawell trajectories, in which case $g \leq 0$. The trajectories in different wells are inversely symmetrical and can be obtained by the transformation $Q \rightarrow-Q, P \rightarrow-P$. Their time dependence can be expressed in terms of the Jacobi elliptic functions [38]. For trajectories in the right well in Fig. 4, where $Q>0$, we have

$$
\begin{gathered}
Q(\tau)=\frac{2^{3 / 2}|g|^{1 / 2} \operatorname{dn} \tau^{\prime}}{\kappa_{+}+\kappa_{-} \operatorname{cn} \tau^{\prime}}, \\
P(\tau)=\frac{\kappa_{+} \kappa_{-}|g|^{1 / 4} \operatorname{sn} \tau^{\prime}}{\kappa_{+}+\kappa_{-} \operatorname{cn} \tau^{\prime}} .
\end{gathered}
$$

Here

$$
\kappa_{ \pm}=\left(1+\mu \pm 2|g|^{1 / 2}\right)^{1 / 2}, \quad \tau^{\prime}=2^{3 / 2}|g|^{1 / 4} \tau .
$$

The parameter of the elliptic functions $m_{J}=m_{J}(g)$ is given by Eq. (12). For $\mu<0$ the function $m_{J}(g)$ monotonically decreases with increasing $g$ from $m_{J}=0$ for $g=g_{\text {min }}=-(\mu+1)^{2} / 4$ to $m_{J} \rightarrow-\infty$ for $g \rightarrow 0$. For $\mu>0$ the function $m_{J}(g)$ becomes nonmonotonic. It first increases from $m_{J}=0$ with increasing $g$, but than decreases, goes trough $m_{J}=0$ for $g=-(1-\mu)^{2} / 4$, and goes to $-\infty$ for $g \rightarrow 0$. The Jacobi functions $\operatorname{sn}\left(\tau^{\prime} \mid m_{J}\right)$, $\operatorname{cn}\left(\tau^{\prime} \mid m_{J}\right)$ and $\operatorname{dn}\left(\tau^{\prime} \mid m_{J}\right)$ for $m_{J}<0$ are equal to 
$\left(1-m_{J}\right)^{-1 / 2} \operatorname{sd}\left(\tilde{\tau}^{\prime} \mid \tilde{m}_{J}\right), \operatorname{cd}\left(\tilde{\tau}^{\prime} \mid \tilde{m}_{J}\right)$, and nd $\left(\tilde{\tau}^{\prime} \mid \tilde{m}_{J}\right)$ with $\tilde{m}_{J}=-m_{J} /\left(1-m_{J}\right)$ and $\tilde{\tau}^{\prime}=\left(1-m_{J}\right)^{1 / 2} \tau^{\prime}$ 38].

The double periodicity of the functions $Q(\tau), P(\tau)$ discussed in Sec. $1 \mathrm{~B}$ is a consequence of the double periodicity of elliptic functions. The expressions for the periods $\tau_{p}^{(1,2)}$, Eq. (11), follow from Eq. (A1). The trajectories in the left well of $g(P, Q)$ in Fig. 4 where $Q<0$, can be written in the form

$$
\begin{aligned}
& Q_{l}(\tau)=Q\left(\tau+\left(\tau_{p}^{(1)}+\tau_{p}^{(2)}\right) / 2\right), \\
& P_{l}(\tau)=P\left(\tau+\left(\tau_{p}^{(1)}+\tau_{p}^{(2)}\right) / 2\right) .
\end{aligned}
$$

This expression shows how to make a transition from one well to another by moving in complex time, which simplifies the analysis of oscillator tunneling.

We note that the function $\mathrm{cn} \tau^{\prime}$ in the expressions for $Q, P$ [A1 has periods $\tau_{p}^{(1)},\left(\tau_{p}^{(1)}+\tau_{p}^{(2)}\right) / 2$ [38] as a function of $\tau$. It's period parallelogram is shown in Fig. 5. In this parallelogram $\mathrm{cn} \tau^{\prime}$ takes on any value twice. Therefore both $Q(\tau)$ and $P(\tau)$ have two poles located at $\tau=\tau_{*}, \tau_{* *}$. The values of $\tau_{*}, \tau_{* *}$ are given by the equation

$$
\begin{aligned}
& \operatorname{cn}\left(2^{3 / 2}|g|^{1 / 4} \tau\right)=-\kappa_{+} / \kappa_{-}, \\
& \tau_{* *}=\frac{1}{2}\left(3 \tau_{p}^{(1)}+\tau_{p}^{(2)}\right)-\tau_{*} .
\end{aligned}
$$

The positions of the poles are shown in Fig. 5 for $m_{J}>0$ and $m_{J}<0$, respectively, with $\phi_{*}=2 \pi \tau_{*} / \tau_{p}^{(1)}, \phi_{* *}=$ $2 \pi \tau_{* *} / \tau_{p}^{(2)}$. For concreteness we choose

$$
\operatorname{Im} \tau_{*}<\operatorname{Im} \tau_{p}^{(2)} / 4
$$

Using the relations between the Jacobi elliptic functions [38] we find that, near the pole at $\tau=\tau_{*}$,

$$
P-i Q \propto-\left(\tau-\tau_{*}\right)^{-1},
$$

whereas $P-i Q$ is not singular at $\tau=\tau_{* *}$. Eq. A6 was used to obtain the explicit form of the matrix element of the operator $\hat{a}=(2 \lambda)^{-1 / 2}(P-i Q)$ in Sec. II C.

\section{APPENDIX B: THE CLASSICAL LIMIT}

In this Appendix we calculate the effective inverse temperature $R^{\prime}(g)$ in the limit of large oscillator Planck num- ber $\bar{n} \gg 1$. The explicit form of the coefficients in Eq. (27) for $R^{\prime}$ follows from the general expression (22) for the transition rates $W_{n n^{\prime}}$,

$$
\begin{array}{r}
\sum_{m} m W_{n+m n}=\sum_{m=-\infty}^{\infty} m\left|a_{-m}\left(g_{n}\right)\right|^{2} \\
\sum_{m} m^{2} W_{n+m n}=(2 \bar{n}+1) \sum_{m=-\infty}^{\infty} m^{2}\left|a_{-m}\left(g_{n}\right)\right|^{2} .
\end{array}
$$

The semiclassical matrix elements $a_{-m}$ are given by Eq. (15). They are Fourier components of the function $a(\phi ; g)$ on the classical trajectory with given $g=g_{n}$.

Using the completeness condition $\sum_{m=-\infty}^{\infty} e^{i m\left(\phi_{2}-\phi_{1}\right)}=2 \pi \delta\left(\phi_{2}-\phi_{1}\right)$ we can rewrite

$$
\begin{gathered}
\sum_{m=-\infty}^{\infty} m\left|a_{-m}(g)\right|^{2}=\frac{1}{2 i \pi} \int_{0}^{2 \pi} d \phi a(\phi ; g) \partial_{\phi} a^{*}(\phi ; g), \\
\sum_{m=-\infty}^{\infty} m^{2}\left|a_{-m}(g)\right|^{2}=(2 \pi)^{-1} \\
\quad \times \int_{0}^{2 \pi} d \phi \partial_{\phi} a(\phi ; g) \partial_{\phi} a^{*}(\phi ; g),
\end{gathered}
$$

with $a(\phi ; g)=(2 \lambda)^{-1 / 2}(P(\tau ; g)-i Q(\tau ; g))$ and $\phi=$ $\omega(g) \tau$. The integrals over $\phi$ can be written as contour integrals over $d P, d Q$ along the trajectories with given $g$. The contour integrals can be further simplified using the Stokes theorem. This gives

$$
\begin{aligned}
\sum_{m=-\infty}^{\infty} m\left|a_{-m}(g)\right|^{2} & \equiv \frac{1}{2 \lambda \pi} M(g) \\
\sum_{m=-\infty}^{\infty} m^{2}\left|a_{-m}(g)\right|^{2} & \equiv \frac{\omega^{-1}(g)}{2 \lambda \pi} N(g)
\end{aligned}
$$

where the functions $M(g)$ and $N(g)$ are given by Eq. (28).
[1] I. Affleck, Phys. Rev. Lett. 46, 388 (1981).

[2] H. Grabert and U. Weiss, Phys. Rev. Lett. 53, 1787 (1984).

[3] A. I. Larkin and Y. N. Ovchinnikov, J. Stat. Phys. 41, 425 (1985).

[4] R. Landauer, J. Appl. Phys. 33, 2209 (1962).
[5] A. D. Ventcel' and M. I. Freidlin, Usp. Mat. Nauk 25, 3 (1970).

[6] M. I. Dykman and M. A. Krivoglaz, Zh. Eksp. Teor. Fiz. 77, 60 (1979).

[7] R. Graham and T. Tél, J. Stat. Phys. 35, 729 (1984).

[8] A. J. Bray and A. J. McKane, Phys. Rev. Lett. 62, 493 
(1989)

[9] M. I. Dykman, Phys. Rev. A 42, 2020 (1990).

[10] R. S. Maier and D. L. Stein, Phys. Rev. E 48, 931 (1993).

[11] R. L. Kautz, Rep. Prog. Phys. 59, 935 (1996).

[12] O. A. Tretiakov, T. Gramespacher, and K. A. Matveev, Phys. Rev. B 67, 073303 (2003).

[13] V. N. Sazonov and V. I. Finkelstein, Doklady Akad. Nauk SSSR 231, 78 (1976).

[14] M. J. Davis and E. J. Heller, J. Chem. Phys. 75, 246 (1981).

[15] A. P. Dmitriev and M. I. Dyakonov, Zh. Eksper. Teor. Fiz. 90, 1430 (1986).

[16] E. J. Heller, J. Phys. Chem. A 103, 10433 (1999).

[17] M. I. Dykman and V. N. Smelyansky, Zh. Eksp. Teor. Fiz. 94, 61 (1988).

[18] L. D. Landau and E. M. Lifshitz, Mechanics, 3rd ed. (Elsevier, Amsterdam, 2004)

[19] R. E. Slusher, L. W. Hollberg, B. Yurke, J. C. Mertz, and J. F. Valley, Phys. Rev. Lett. 55, 2409 (1985).

[20] L.-A. Wu, H. J. Kimble, J. L. Hall, and H. Wu, Phys. Rev. Lett. 57, 2520 (1986).

[21] R. Loudon and P. L. Knight, J. Mod. Opt. 34, 709 (1987).

[22] G. A. Garrett, A. G. Rojo, A. K. Sood, J. F. Whitaker, and R. Merlin, Science 275, 1638 (1997).

[23] D. Rugar and P. Grutter, Phys. Rev. Lett. 67, 699 (1991).

[24] V. Natarajan, F. Difilippo, and D. E. Pritchard, Phys. Rev. Lett. 74, 2855 (1995).

[25] I. Siddiqi, R. Vijay, F. Pierre, C. M. Wilson, M. Metcalfe, C. Rigetti, L. Frunzio, and M. H. Devoret, Phys. Rev. Lett. 93, 207002 (2004).

[26] J. Claudon, F. Balestro, F. W. J. Hekking, and O. Buisson, Phys. Rev. Lett. 93, 187003 (2004).

[27] M. Blencowe, Phys. Rep. 395, 159 (2004).

[28] J. S. Aldridge and A. N. Cleland, Phys. Rev. Lett. 94, 156403 (2005).

[29] J. W. F. Woo and R. Landauer, IEEE J. Quant. Electr. QE 7, 435 (1971).

[30] P. D. Drummond, K. J. McNeil, and D. F. Walls, Optica Acta 28, 211 (1981).
[31] M. Wolinsky and H. J. Carmichael, Phys. Rev. Lett. 60, 1836 (1988).

[32] P. D. Drummond and P. Kinsler, Phys. Rev. A 40, 4813 (1989).

[33] P. Kinsler and P. D. Drummond, Phys. Rev. A 43, 6194 (1991).

[34] G. Y. Kryuchkyan and K. V. Kheruntsyan, Opt. Commun. 127, 230 (1996).

[35] J. Tan and G. Gabrielse, Phys. Rev. A 48, 3105 (1993).

[36] L. J. Lapidus, D. Enzer, and G. Gabrielse, Phys. Rev. Lett. 83, 899 (1999).

[37] M. I. Dykman, C. M. Maloney, V. N. Smelyanskiy, and M. Silverstein, Phys. Rev. E 57, 5202 (1998).

[38] M. Abramowitz and I. A. Stegun, eds., Handbook of Mathematical Functions with Formulas, Graphs, and Mathematical Table (Dover, Oxford, 1974).

[39] L. D. Landau and E. M. Lifshitz, Quantum mechanics. Non-relativistic theory, 3rd ed. (ButterworthHeinemann, Oxford, 1981)

[40] E. Knobloch and K. A. Wiesenfeld, J. Stat. Phys. 33, 611 (1983).

[41] R. Graham and T. Tél, Phys. Rev. A 35, 1328 (1987).

[42] M. A. Ivanov, L. B. Kvashnina, and M. A. Krivoglaz, Sov. Phys. Solid State,Ussr 7, 1652 (1966).

[43] A. S. Barker and A. J. Sievers, Rev. Mod. Phys. 47, S1 (1975).

[44] M. I. Dykman and M. A. Krivoglaz, Soviet Physics Reviews (Harwood, New York, 1984), vol. 5, pp. 265-441.

[45] W. K. Hensinger, H. Haffer, A. Browaeys, N. R. Heckenberg, K. Helmerson, C. McKenzie, G. J. Milburn, W. D. Phillips, S. L. Rolston, H. Rubinsztein-Dunlop, et al., Nature 412, 52 (2001).

[46] D. A. Steck, W. H. Oskay, and M. G. Raizen, Science 293, 274 (2001).

[47] W. K. Hensinger, N. R. Heckenberg, G. J. Milburn, and H. Rubinsztein-Dunlop, J. Opt. B 5, R83 (2003).

[48] B. Wielinga and G. J. Milburn, Phys. Rev. A 48, 2494 (1993). 